\title{
High Prevalence of Autoimmune Gastritis in Patients with Nonalcoholic Steatohepatitis
}

\author{
Miwa Kawanaka ${ }^{1}$, Tomohiro Tanikawa ${ }^{1}$, Tomoari Kamada ${ }^{2}$, Katusinori Ishii ${ }^{1}$, Noriyo Urata ${ }^{1}$, \\ Jun Nakamura ${ }^{1}$, Ken Nishino ${ }^{1}$, Mitsuhiko Suehiro ${ }^{1}$, Takako Sasai ${ }^{1}$, Noriaki Manabe ${ }^{3}$, \\ Yasumasa Monobe ${ }^{4}$, Hirofumi Kawamoto ${ }^{1}$ and Ken Haruma ${ }^{1}$
}

\begin{abstract}
:
Objective To evaluate the prevalence of autoimmune gastritis in patients with histologically proven nonalcoholic steatohepatitis (NASH).

Methods A total of 33 patients with NASH and 143 patients with chronic liver disease $(66,24,22,10,1$, and 21 patients with hepatitis $\mathrm{C}$, hepatitis $\mathrm{B}$, autoimmune hepatitis/primary biliary cholangitis, non-B/non-C hepatitis, fatty liver, and alcoholic disease, respectively) who underwent upper gastrointestinal endoscopy between January 2013 and August 2016 were retrospectively assessed to determine the prevalence of autoimmune gastritis. The clinical characteristics of these patients with NASH and autoimmune gastritis were examined, and the clinical characteristic and biomarkers were compared between patients with NASH with and without autoimmune gastritis.

Results Six of the 33 patients with NASH (19.4\%) were diagnosed with autoimmune gastritis. The prevalence of autoimmune gastritis was higher in patients with NASH than in those with other chronic liver diseases [4/143 (2.8\%), $\mathrm{p}=0.002]$. All six patients with NASH and autoimmune gastritis exhibited high serum gastrin levels; five of the patients were positive for anti-parietal cell antibodies, and one was negative for anti-parietal cell antibodies but positive for intrinsic factor antibody. Furthermore, 1 patient presented with iron-deficiency anemia (hemoglobin $<11 \mathrm{~g} / \mathrm{dL}$ ), but none developed pernicious anemia. Endocrine cell micronests were found in four patients. Patients with NASH and autoimmune gastritis tended to be older with lower ferritin levels than the other patients.

Conclusion The prevalence of NASH with concomitant autoimmune gastritis was high, highlighting the need for upper endoscopy for the diagnosis of autoimmune gastritis and gastric malignancies.
\end{abstract}

Key words: nonalcoholic steatohepatitis, autoimmune gastritis, gastrointestinal endoscopy, gastrin

(Intern Med 58: 2907-2913, 2019)

(DOI: 10.2169/internalmedicine.2693-19)

\section{Introduction}

Autoimmune gastritis, also known as type A gastritis, is characterized by atrophic changes in the fundic mucosa caused by autoimmune mechanisms, such as anti-parietal cell antibodies, that lead to hypergastrinemia and hyperplasia of enterochromaffin-like cells, resulting in complications like hyperplastic polyps, carcinoid tumors, and gastric cancer. Typically, autoimmune gastritis is diagnosed during the medical evaluation of patients with pernicious anemia or type-1 neuroendocrine tumors (NETs) and may be associated with autoimmune thyroid disease or type 1 diabetes mellitus (DM) (1-9). No data are available on the prevalence of autoimmune gastritis, which is asymptomatic in the initial stage. In the late stage, autoimmune gastritis may cause per-

${ }^{1}$ Department of General Internal Medicine, Kawasaki Medical School, Japan, ${ }^{2}$ Department of Health Care Medicine, Kawasaki Medical School, Japan, ${ }^{3}$ Department of Clinical Pathology and Laboratory, Kawasaki Medical School, Japan and ${ }^{4}$ Department of Pathology, Kawasaki Medical School, Japan

Received: January 17, 2019; Accepted: May 12, 2019; Advance Publication by J-STAGE: July 10, 2019

Correspondence to Dr. Ken Haruma, kharuma@med.kawasaki-m.ac.jp 
nicious anemia characterized by megaloblastic anemia that can be diagnosed in clinical practice. In Japan, autoimmune gastritis is considered to be a rare disease (10). In a clinical statistical analysis, Sugihara and Yawata estimated that the prevalence of pernicious anemia caused by autoimmune gastritis was $0.34-0.5$ per 100,000 persons in the Japanese population (11).

Recently, nonalcoholic steatohepatitis (NASH) has been classified as a part of the spectrum of nonalcoholic fatty liver diseases (NAFLD) associated with metabolic syndrome, and the prevalence of NASH has been rapidly increasing worldwide (12). However, the pathogenesis of NASH is not completely understood. Insulin resistance, inflammatory cytokines, and oxidative stress may play important roles in the development and progression of NASH (13-15).

At our institution, we diagnosed autoimmune gastritis in six patients with NASH on the basis of an endoscopic examination between January 2013 and August 2016. Given that autoimmune gastritis is considered a rare presentation, we investigated the association between autoimmune gastritis and NASH in this retrospective study.

\section{Materials and Methods}

\section{Study population}

A total of 1,031 consecutive outpatients $\geq 30$ years of age who underwent esophagogastroduodenal endoscopy at General Medical Center, Kawasaki Medical Hospital between January 2013 and August 2016 were eligible for this study. We retrospectively reviewed the endoscopic findings and laboratory data of 33 patients with NASH, which was confirmed via a liver biopsy; these patients underwent upper gastrointestinal endoscopy for the diagnosis of organic diseases or as part of a healthcare examination at the outpatient clinic.

In this retrospective cross-sectional study, we compared 33 patients with NASH and 144 patients with chronic liver diseases, including 66, 24, 22, 10, 1, and 21 patients with hepatitis $\mathrm{C}$, hepatitis $\mathrm{B}$, autoimmune hepatitis and/or primary biliary cholangitis, non-B/non- $\mathrm{C}$ hepatitis, fatty liver, and alcoholic disease, respectively, who underwent upper gastrointestinal endoscopy between January 2013 and August 2016.

\section{Diagnostic criteria}

In this study, autoimmune gastritis was defined based on the concomitant presence of corpus-predominant atrophic gastritis, fasting hypergastrinemia ( $>245.1 \mathrm{pg} / \mathrm{mL})$, histological confirmation of corpus atrophy, and positivity for antiparietal cell and/or intrinsic factor antibodies, as previously reported $(16,17)$. The exclusion criteria included the use of nonsteroidal anti-inflammatory drugs, antacids, H2-receptor antagonists, proton pump inhibitors, and antibiotics in the month preceding endoscopy; pregnancy; a history of gastric surgery, systemic diseases such as collagen disease, inflammatory bowel disease, and eosinophilic gastroenteritis; and a history of treatment for Helicobacter pylori. Patients with corpus-predominant atrophic gastritis diagnosed by endoscopic findings were tested for gastric autoantibodies, fasting serum gastrin, and $H$. pylori antibody.

A NASH diagnosis was based on the following criteria: (i) alcohol intake $\leq 20 \mathrm{~g} /$ day in women and $\leq 30 \mathrm{~g} /$ day in men; (ii) absence of detectable hepatitis B surface antigen or hepatitis C virus RNA, autoimmune liver disease, druginduced liver injury, or metabolic liver disease such as Wilson's disease and hemochromatosis; and (iii) presence of steatosis $(>5 \%)$, steatohepatitis, and inflammation, and hepatocellular ballooning. The liver biopsy findings were evaluated by two expert pathologists, and the features were graded as follows using the NAFLD activity score system proposed by the NASH Clinical Research Network: lobular inflammation (0-3), steatosis (0-3), and hepatocellular ballooning (0-2). The fibrosis stage was assessed according to Brunt's classification $(18,19)$.

The study protocol was in accordance with the 1975 Declaration of Helsinki and approved by the research ethics committee of the study institution. The requirement for informed consent was waived by the research ethics committee due to the retrospective study design.

\section{Statistical analyses}

Continuous variables at baseline were expressed as the mean with the standard deviation. Comparisons between two groups were performed using Student's $t$-test for continuous variables and the $\chi^{2}$ test for categorical variables. A $\mathrm{p}<0.05$ was considered statistically significant. All analyses were performed using JMP software version 13.2 (SAS Institute Japan).

\section{Results}

In total, 6 (19.4\%; 3 women and 3 men; mean age, 75.3 years) of the 33 patients with NASH were diagnosed with autoimmune gastritis. The prevalence of autoimmune gastritis was significantly higher in patients with NASH than those with other chronic liver diseases $(19.4 \%$ vs. $2.8 \%, \mathrm{p}=$ 0.002 ). An analysis of the patient background characteristics revealed no marked differences in the age or sex between the two groups. However, the body mass index, serum aspartate aminotransferase, cholesterol, triglyceride, hemoglobin A1c, serum iron, ferritin, and platelet count were higher in patients with NASH than in those with other chronic liver diseases (Table 1).

Table 2 summarizes the characteristics of the six patients with NASH who developed autoimmune gastritis. All patients showed high serum gastrin levels $(>1,500 \mathrm{pg} / \mathrm{mL}$ in 5 of the 6 patients). In addition, five patients were positive for anti-parietal cell antibodies (PCAs), whereas one patient who was negative for PCAs was positive for intrinsic factor antibodies. One patient presented with iron-deficiency ane- 
Table 1. Clinical Characteristics of the Study Patients and Biomarkers for NASH and Chronic Liver Disease.

\begin{tabular}{lccc}
\hline \multicolumn{1}{c}{ Characteristics } & NASH & Chronic liver disease & p value \\
\hline Frequency of autoimmune gastritis & $6 / 33(19.4 \%)$ & $4 / 143(2.8 \%)$ & 0.0026 \\
Age & $65.1 \pm 11.9$ & $67.6 \pm 10.8$ & 0.2783 \\
Sex, male & $42 \%$ & $55 \%$ & 0.1784 \\
BMI $\left(\mathrm{kg} / \mathrm{m}^{2}\right)$ & $27.6 \pm 4.0$ & $23.2 \pm 3.5$ & $<0.0001$ \\
Liver cirrhosis & $6.50 \%$ & $54 \%$ & $<0.0001$ \\
ALT (IU/L) & $52.0 \pm 45.5$ & $26.8 \pm 30.3$ & 0.3202 \\
AST (IU/L) & $43.1 \pm 29.7$ & $33.9 \pm 21.7$ & $<0.0001$ \\
$\gamma$-GTP $(\mathrm{IU} / \mathrm{L})$ & $60.1 \pm 72.6$ & $62.4 \pm 95.5$ & 0.5408 \\
Total bilirubin $(\mathrm{mg} / \mathrm{dL})$ & $0.83 \pm 0.4$ & $1.0 \pm 0.7$ & 0.0748 \\
Total cholesterol $(\mathrm{ng} / \mathrm{dL})$ & $197 \pm 40$ & $177 \pm 43$ & 0.0239 \\
Triglyceride $(\mathrm{ng} / \mathrm{dL})$ & $165.4 \pm 75.4$ & $117.4 \pm 72.0$ & $<0.0001$ \\
LDL-C (ng/dL) & $119.4 \pm 40.8$ & $104.8 \pm 33.7$ & 0.0757 \\
HDL-C (ng/dL) & $48.3 \pm 20.2$ & $53.5 \pm 16.7$ & 0.0259 \\
Platelet count $(104 / \mu \mathrm{g})$ & $19.4 \pm 6.6$ & $15.5 \pm 7.1$ & 0.0021 \\
Hemoglobin $(\mathrm{g} / \mathrm{dL})$ & $13.8 \pm 1.5$ & $12.9 \pm 2.5$ & 0.0713 \\
Hemoglobin Alc & $5.9 \pm 0.7$ & $5.4 \pm 0.7$ & 0.003 \\
Iron $(\mu \mathrm{g} / \mathrm{dL})$ & $122 \pm 49.3$ & $84.7 \pm 64.3$ & 0.0116 \\
Ferritin $(\mathrm{ng} / \mathrm{dL})$ & $192.4 \pm 283$ & $95.7 \pm 128$ & 0.0319 \\
$\gamma$-Globulin $(\%)$ & $17.7 \pm 4.4$ & $20.2 \pm 5.8$ & 0.1175 \\
\hline
\end{tabular}

NASH: nonalcoholic steatohepatitis, BMI: body mass index, ALT: alanine aminotransferase, AST: aspartate aminotransferase, $\gamma$-GTP: gamma glutamyl transpeptidase, LDL-C: low-density lipoprotein cholesterol, HDL-C: high-density lipoprotein cholesterol

Table 2. Clinical Characteristics of the Patients with NASH who Developed Autoimmune Gastritis (n=6).

\begin{tabular}{ccccccccccccc}
\hline & $\begin{array}{c}\text { Age, } \\
\text { sex }\end{array}$ & $\begin{array}{c}\text { Gastrin } \\
(\mathrm{pg} / \mathrm{mL})\end{array}$ & ECM & $\begin{array}{c}\text { PCA } \\
(\text { Dilution } \\
\text { rate })\end{array}$ & $\begin{array}{c}\text { PGI } \\
(\mathrm{ng} / \mathrm{mL})\end{array}$ & $\begin{array}{c}\text { PGII } \\
(\mathrm{ng} / \mathrm{mL})\end{array}$ & $\begin{array}{c}\text { PGI/ } \\
\text { PGII }\end{array}$ & IFA & $\begin{array}{c}\text { B12 } \\
(\mathrm{pg} / \mathrm{mL})\end{array}$ & $\begin{array}{c}\text { Folic } \\
\text { acid } \\
(\mathrm{ng} / \mathrm{mL})\end{array}$ & $\begin{array}{c}\text { Hemoglobin } \\
(\mathrm{g} / \mathrm{dL})\end{array}$ & $\begin{array}{c}\text { Helicobacter } \\
\text { pylori } \\
\text { antibody }\end{array}$ \\
\hline 1 & $80 \mathrm{~F}$ & 5,254 & + & 20 & 4.3 & 6 & 0.7 & + & 252 & 148 & 14.3 & + \\
2 & $62 \mathrm{~F}$ & 4,962 & + & 80 & 2 & 7.4 & 0.38 & - & 362 & 10.6 & 10.8 & - \\
3 & $83 \mathrm{~F}$ & 7,800 & + & Negative & 6.9 & 10.1 & 0.7 & + & 176 & 21.2 & 11.7 & - \\
4 & $75 \mathrm{M}$ & 2,368 & No biopsy & 10 & 5.2 & 11.4 & 0.5 & - & 111 & 8.8 & 14.9 & + \\
5 & $57 \mathrm{M}$ & 249 & No biopsy & 10 & 15 & 3.5 & 4.3 & - & 486 & 8.1 & 13.7 & + \\
6 & $84 \mathrm{M}$ & 1,641 & + & 10 & 76.4 & 10.4 & 7.3 & - & 890 & 7.9 & 11.8 & - \\
\hline
\end{tabular}

NASH: nonalcoholic steatohepatitis, ECM: endocrine cell micronest, PCA: anti-parietal cell antibody, PGI: pepsinogen I, PGII: pepsinogen II, IFA: intrinsic factor antibody, M: male, F: female

mia (hemoglobin $<11 \mathrm{~g} / \mathrm{dL}$ ), but none of the patients developed pernicious anemia. Endocrine cell micronests (ECMs) were found in 4 patients, and $H$. pylori infection was observed in $3(50 \%)$ patients. Although two patients were positive for anti-thyroglobulin antibodies, none of the patients required treatment for thyroid disease. There were 2, 2, and 1 patient with stage 1,3 , and $4 \mathrm{NASH}$, respectively, among the six patients with autoimmune gastritis. Furthermore, the NASH patients with autoimmune gastritis tended to be older with significantly lower serum ferritin levels than those without autoimmune gastritis. However, no significant differences were observed in other patient characteristics between NASH patients with and without autoimmune gastritis (Table 3 ).

Case 1 is described below to illustrate NASH with autoimmune gastritis. A histological examination of the transcu- taneous liver biopsy sample after hematoxylin/eosin and Azan staining revealed lobular inflammation, hepatocellular ballooning degeneration, and perisinusoidal fibrosis as well as the presence of macrovesicular hepatocellular steatosis. Consequently, the patient was diagnosed with NASH (Brunt's classification: stage 1, grade 1) (Fig. 1). An endoscopic examination revealed typical findings of corpuspredominant atrophic gastritis (Fig. 2). The biopsy specimens showed mild inflammation and severe atrophy in the corpus mucosa. However, no inflammation or atrophy was observed in the pyloric mucosa. In Fig. 3, the upper right panel shows the presence of several ECMs in the corpus mucosa, and the lower right panel shows positive chromogranin staining. 
Table 3. Clinical Characteristics and Biomarkers of Patients with NASH with and without Autoimmune Gastritis.

\begin{tabular}{|c|c|c|c|}
\hline Characteristics & Autoimmune gastritis (+) & Autoimmune gastritis (-) & $\mathrm{p}$ value \\
\hline Age & $73.5 \pm 11.0$ & $63.1 \pm 11.4$ & 0.0426 \\
\hline Sex, male & $66 \%$ & $60 \%$ & 0.7616 \\
\hline BMI $\left(\mathrm{kg} / \mathrm{m}^{2}\right)$ & $26.4 \pm 2.1$ & $27.8 \pm 4.3$ & 0.7451 \\
\hline Stage $(0 / 1 / 2 / 3 / 4)$ & $0 / 2 / 0 / 3 / 1$ & $1 / 5 / 4 / 10 / 5$ & 0.6207 \\
\hline Grade $(0 / 1 / 2 / 3)$ & $0 / 1 / 4 / 1$ & $0 / 11 / 12 / 2$ & 0.4139 \\
\hline Diabetes mellitus & $50 \%$ & $56 \%$ & 0.7912 \\
\hline Hypertension & $33.30 \%$ & $52 \%$ & 0.4071 \\
\hline Dyslipidemia & $100 \%$ & $92.00 \%$ & 0.3447 \\
\hline ALT (IU/L) & $33.6 \pm 12.5$ & $49.5 \pm 30.9$ & 0.4092 \\
\hline AST (IU/L) & $41.1 \pm 15.0$ & $38.4 \pm 15.6$ & 0.745 \\
\hline$\gamma-\mathrm{GTP}(\mathrm{IU} / \mathrm{L})$ & $42.3 \pm 18.0$ & $59.1 \pm 71.0$ & 0.7075 \\
\hline Total cholesterol (ng/dL) & $201 \pm 22.8$ & $197 \pm 43.5$ & 0.617 \\
\hline Platelet count $\left(10^{4} / \mu \mathrm{g}\right)$ & $20.2 \pm 7.4$ & $19.4 \pm 6.5$ & 0.7451 \\
\hline Hemoglobin $(\mathrm{g} / \mathrm{dL})$ & $13.1 \pm 1.7$ & $14.0 \pm 1.5$ & 0.3468 \\
\hline HOMA-IR & $2.4 \pm 0.9$ & $4.3 \pm 2.6$ & 0.126 \\
\hline Iron $(\mu \mathrm{g} / \mathrm{dL})$ & $114 \pm 60$ & $124 \pm 47$ & 0.7754 \\
\hline Ferritin (ng/dL) & $48.6 \pm 50.8$ & $228 \pm 306$ & 0.0076 \\
\hline$\gamma$-Globulin & $16.4 \pm 2.5$ & $18.1 \pm 5.0$ & 0.6015 \\
\hline Antinuclear antibody & $16 \%$ & $25 \%$ & 0.6567 \\
\hline Leptin $(\mathrm{ng} / \mathrm{dL})$ & $11.4 \pm 5.8$ & $13.5 \pm 8.4$ & 0.824 \\
\hline Adiponectin $(\mu \mathrm{g} / \mathrm{mL})$ & $6.2 \pm 1.8$ & $6.1 \pm 2.3$ & 1 \\
\hline High-sensitivity CRP (mg/dL) & $0.16 \pm 0.1$ & $0.13 \pm 0.15$ & 0.2299 \\
\hline WFA+M2BP (C.O.I) & $1.3 \pm 0.9$ & $1.6 \pm 0.9$ & 0.5711 \\
\hline Type-4 collagen $7 \mathrm{~S}(\mathrm{ng} / \mathrm{mL})$ & $4.8 \pm 1.0$ & $4.9 \pm 2.1$ & 0.8623 \\
\hline
\end{tabular}

NASH: nonalcoholic steatohepatitis, NAFLD: nonalcoholic fatty liver disease, BMI: body mass index, ALT: alanine aminotransferase, AST: aspartate aminotransferase, $\gamma$-GTP: gamma glutamyl transpeptidase, HOMA-IR: homeostatic model assessment-insulin resistance, CRP: C-reactive protein, WFA+M2BP: Wisteria floribunda agglutinin Mac-2 Binding protein

\section{Discussion}

This is the first study to report a positive association between NASH and autoimmune gastritis, which is a critical condition because it causes hypergastrinemia and can lead to pernicious anemia, gastric cancer, and gastric NETs.

In the current retrospective cross-sectional study, the presence of autoimmune gastritis was determined by assessing endoscopic images obtained from 177 patients with chronic liver disease who underwent upper gastrointestinal endoscopy. Initially, autoimmune gastritis was suspected based on endoscopic findings of corpus-predominant atrophic gastritis, which was diagnosed from the presence of ECMs and the results of testing for anti-parietal cell and anti-intrinsic factor antibodies, serum gastrin, and gastric tissue samples. Autoimmune gastritis was defined as the presence of corpuspredominant atrophic gastritis with hypergastrinemia in the presence of anti-PCAs and/or ECMs in the corpus mucosa.

One patient in the current study cohort presented with iron-deficiency anemia. Achlorhydria as a result of autoimmune gastritis is associated with iron-deficiency anemia. Thus, the recognition of autoimmune gastritis is important in patients with refractory iron-deficiency anemia $(20,21)$.

Autoimmune gastritis may be associated with autoimmune thyroid disease or type 1 DM. However, an association between autoimmune gastritis and NASH was not reported previously. Kutsuma et al. reported that overweight Japanese men had low pepsinogen I levels and a low pepsinogen I/II ratio (22). These intriguing findings may be applicable to not only patients with type A gastritis but also those with $H$. pylori-positive type B gastritis. A study in northern Sweden found that atrophic corpus gastritis was more common among obese patients (body mass index $\geq 30 \mathrm{~kg} / \mathrm{m}^{2}$ ) and in patients with DM, particularly men 35-44 years of age (23). NASH may be associated with autoimmune gastritis, as it is common in patients with obesity and DM. However, the underlying mechanism is not completely clear. NASH can emerge in the presence of various clinical conditions, including insulin resistance and oxidative stress as well as other conditions that affect iron levels and alter intestinal bacterial flora $(13-15,24,25)$. In some patients, autoimmune factors may cause NASH. Furthermore, in autoimmune gastritis, the barrier of the gastric wall or the intestines is impaired because of decreased gastric acid levels, which alters the intestinal bacterial flora and causes leaky gut syndrome (26), thereby promoting the flow of endotoxins into the portal vein and eventually leading to NASH.

The diagnosis of autoimmune gastritis is challenging prior to the onset of anemia, which occurs in advanced-stage dis- 


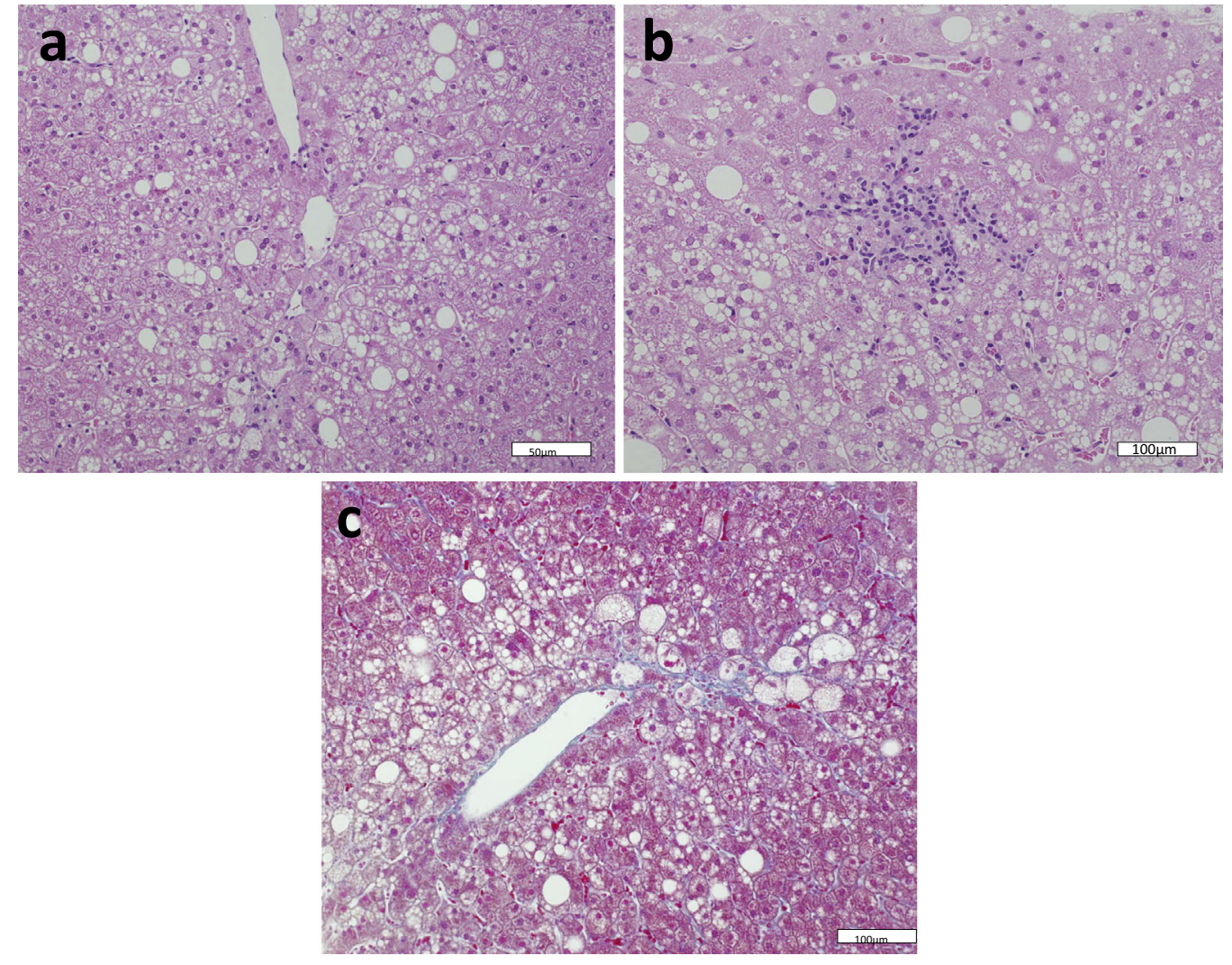

Figure 1. Histological examination of transcutaneous liver biopsy after Hematoxylin and Eosin staining $(a, \times 10)(b, \times 20)$ and Azan $(c, \times 20)$ staining showing lobular inflammation, hepatocellular ballooning degeneration, and perisinusoidal fibrosis as well as the presence of macrovesicular hepatocellular steatosis. Consequently, the patient was diagnosed with nonalcoholic steatohepatitis (NASH; Brunt's classification, stage 1, grade 1).
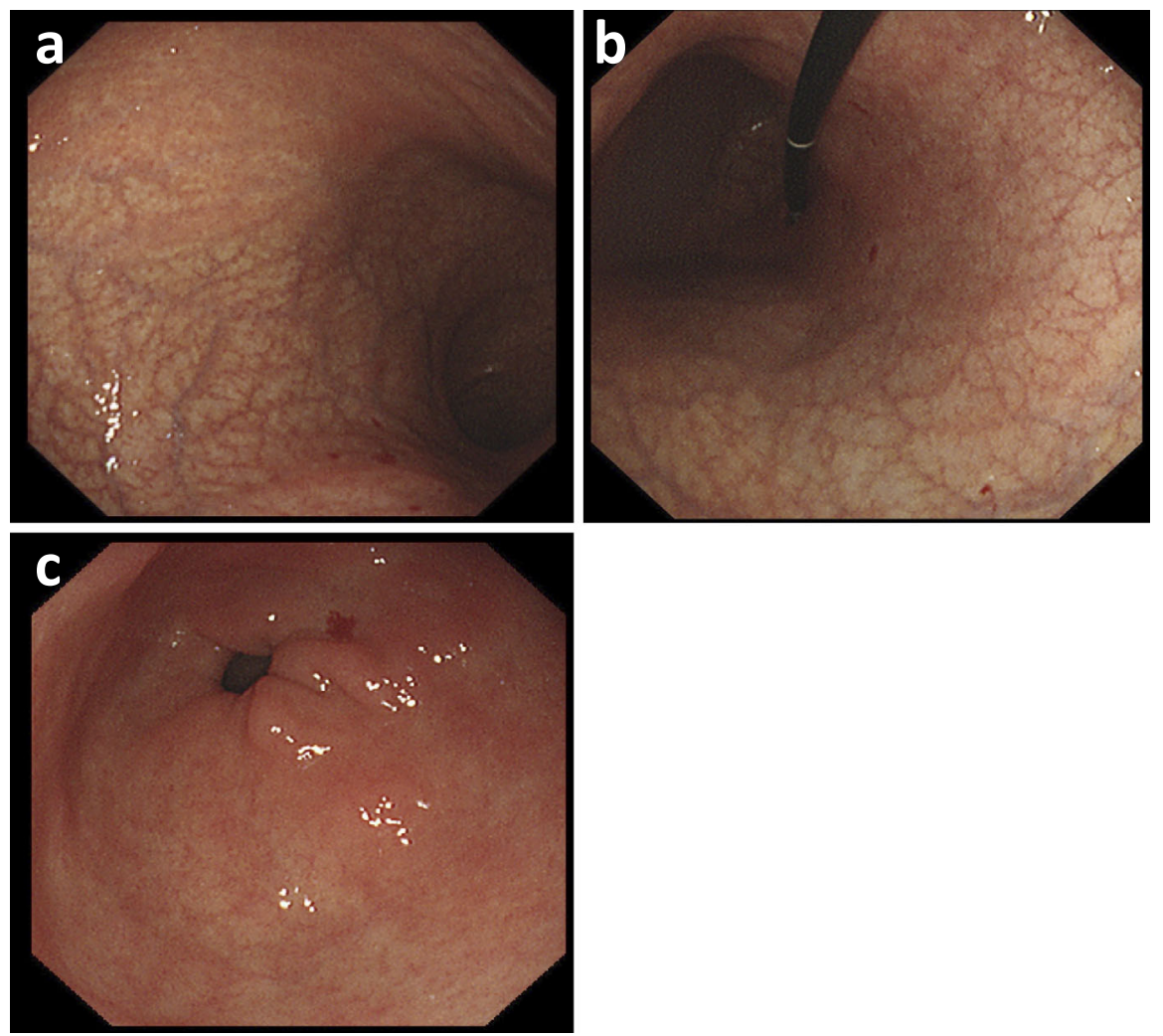

Figure 2. Endoscopic examination reveals typical endoscopic findings of corpus-predominant atrophic gastritis (a and b, corpus; c, antrum). 

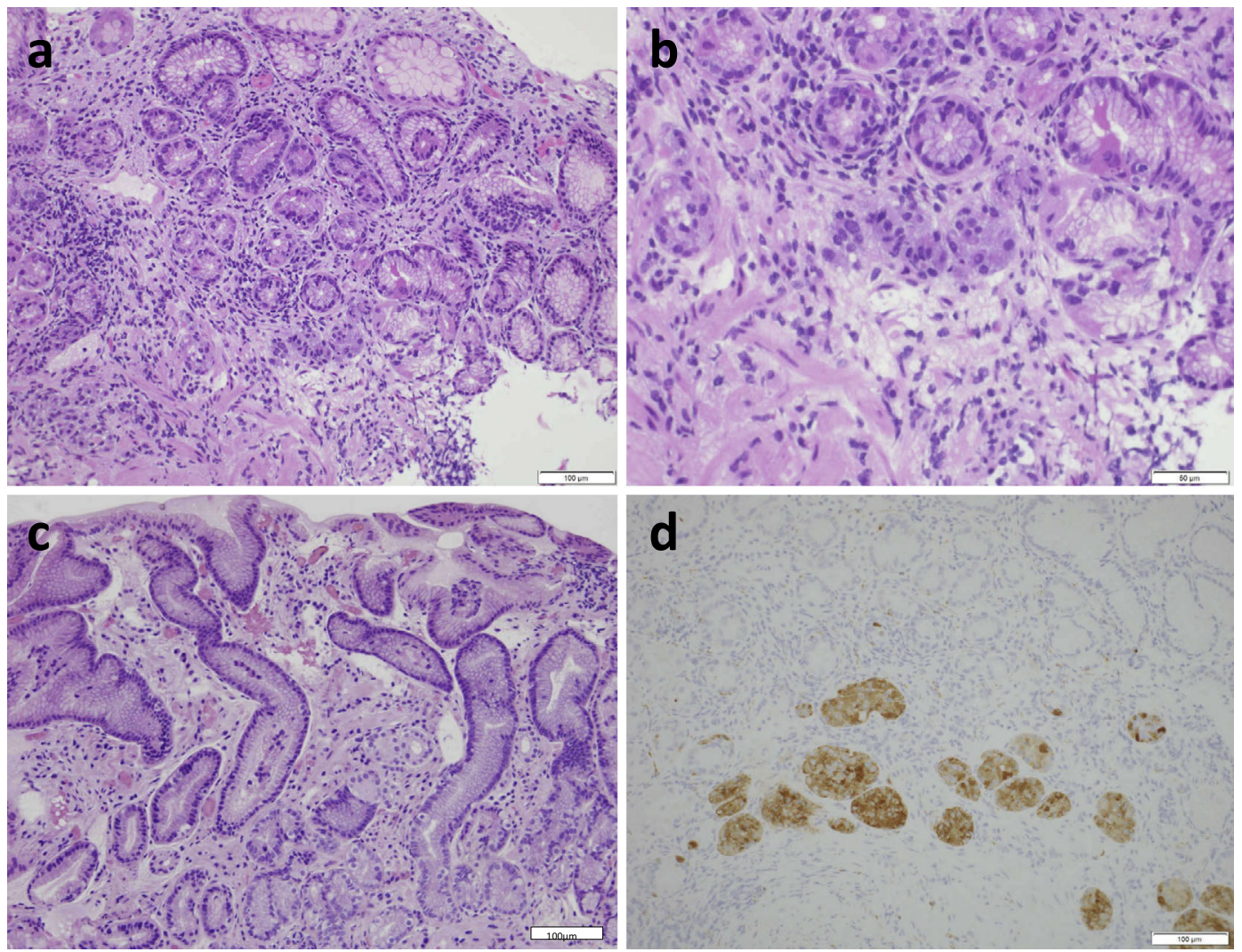

Figure 3. Histological examination reveals mild inflammation and severe atrophy in the corpus mucosa $(a, \times 40)$. Several ECMs are noted in the corpus mucosa $(b, \times 40)$, but the pyloric mucosa appears normal $(c, \times 40)$. In another specimen of the corpus mucosa, ECMs are stained by chromogranin A $(\mathbf{d}, \times \mathbf{4 0})$.

ease, unless autoimmune gastritis is suspected based on reversed atrophic-type gastritis on upper endoscopy or altered gastrin levels. In the current study, the patients with NASH were older and had lower ferritin levels, which are usually elevated in NASH, than those without NASH (27). Thus, autoimmune gastritis in patients with NASH can be screened by measuring gastrin and ferritin levels. The present study is limited by the small number of cases and poor understanding of the underlying pathogenesis.

\section{Conclusion}

Autoimmune gastritis is common in patients with NASH. The rapid increase in the prevalence of NASH in Japan might lead to an associated increase in the incidence of autoimmune gastritis. The early diagnosis of autoimmune gastritis may prevent pernicious anemia due to vitamin B12 deficiency or peripheral neuropathy and may help identify patients at high risk for gastric cancer or NETs.

The authors state that they have no Conflict of Interest (COI).

\section{Acknowledgement}

The authors wish to thank Dr. Keisuke Hino at Kawasaki Medical School and Mrs. Miki Noguchi for their support and assistance in the preparation of the manuscript.

\section{References}

1. Strickland RG, Mackar IR. A reappraisal of the nature and significance of chronic atrophic gastritis. Am J Dig Dis 18: 426-440, 1973.

2. Stockbrugger RW, Menon GG, Beilby JO, Mason RR, Cotton PB. Gastroscopic screening in 80 patients with pernicious anemia. Gut 24: 1141-1147, 1983.

3. Lam-Tse WK, Batstra MR, Koeleman BP, et al. The association between autoimmune thyroiditis, autoimmune gastritis and type 1 diabetes. Pediatr Endocrinol Rev 10: 529-541, 2013.

4. Minalyan A, Benhammou JN, Artashesyan A, Lewis MS, Pisegna JR. Autoimmune atrophic gastritis: current perspectives. Clin Exp Gastroenterol 10: 19-27, 2017.

5. Irvine WJ. The association of atrophic gastritis with autoimmune thyroid disease. Clin Endocrinol Metab 4: 351-377, 1975.

6. Whittingham S, Youngchaiyud U, Mackay IR, Buckley JD, Morris PJ. Thyrogastric autoimmune disease. Studies on the cell-mediated immune system and histocompatibility antigens. Clin Exp Immunol 19: 289-299, 1975.

7. Murphy G, Dawsey SM, Engels EA, et al. Cancer risk after pernicious anemia in the US elderly population. Clin Gastroenterol Hepatol 13: 2282-2289, 2015.

8. Park JY, Cornish TC, Lam-Himlin D, Shi C, Monotgomery E. Gastric lesions in patients with autoimmune metaplastic atrophic gastritis (AMAG) in a tertiary care setting. Am J Surg Pathol 34: 1591-1598, 2010.

9. Zhang H, Nie X, Song Z, Cui R, Jin Z. Hyperplastic polyps arising in autoimmune metaplastic atrophic gastritis patients: is this a distinct clinicopathological entity? Scand J Gastroenterol 53: 
1186-1193, 2018.

10. Haruma K, Komoto K, Kawaguchi H, et al. Pernicious anemia and Helicobacter pylori infection in Japan: evaluation in a country with a prevalence of infection. Am J Gastroenterol 90: 1107-1110, 1995.

11. Sugihara T, Tawata Y. Japanese clinical statistical data of patients with pernicious anemia. Nihon Rinsho 50: 771-786, 1992.

12. Vernon G, Baranova A, Younossi ZM. Systematic review: the epidemiology and natural histology of non-alcoholic fatty liver disease and non-alcoholic steatohepatitis in adults. Aliment Pharmacol Ther 34: 274-285, 2011.

13. Tilg H, Moschen AR. Evolution of inflammation in nonalcoholic fatty liver disease: the multiple parallel hits hypothesis. Hepatology 52: 1836-1846, 2010.

14. Ttuneyama K, Babe H, Kikuchi K, et al. Autoimmune features in metabolic liver disease: a single-center experience and review of the literature. Clinic Rev Allerg Immuno 45: 143-148, 2013.

15. Sutti $S$, Jindal A, Bruzzi $S$, et al. Is there a role for adaptive immunity in nonalcoholic steatohepatitis? World J Hepatol 7: 17251729, 2015.

16. Lahner E, Norman GL, Severi C, et al. Reassessment of intrinsic factor and parietal cell autoantibodies in atrophic gastritis with respect to cobalamin deficiency. Am J Gastroenterol 204: 20712079, 2009.

17. Haruma K, Yoshihara M, Sumii K, et al. Gastric acid secretion, serum pepsinogen I, and serum gastrin in Japanese with gastric hyperplastic polyps or polypoid-type early gastric carcinoma. Scand J Gastroenterol 28: 633-637, 1993.

18. Kleiner DE, Brunt EM, Van Natta M, et al. Design and validation of a histological scoring system for nonalcoholic fatty liver disease. Hepatology 41: 1313-1321, 2005.

19. Brunt EM, Kleiner DE, Wilson LA, Belt P, Neuschwander-Tetri BA; NASH Clinical Research Network (CRN). Nonalcoholic fatty liver disease (NAFLD) activity score and the histologic diagnosis in NAFLD: distinct clinicopathologic meanings. Hepatology 53: 810-820, 2011.

20. Hershko C, Hoffbrand AV, Keret D, et al. Role of autoimmune gastritis, Helicobacter pylori and celiac disease in refractory or unexplained iron deficiency anemia. Haematologica 90: 585-595, 2005.

21. Carabotti M, Lahner E, Esposita G, et al. Upper gastrointestinal symptoms in autoimmune gastritis. A cross-sectional study. Medicine 96: e5784, 2017.

22. Kutsuma A, Oshida H, Suwa K, Nakajima K. A possible association of low pepsinogen 1 and pepsinogen $1 / 2$ with low and high body weight in Japanese men. Clin Biochemistry 47: 126-128, 2014.

23. Song H, Held M, Sandin S, et al. Increase in the prevalence of atrophic gastritis among adults age 35 to 44 years old in northern Sweden between 1990 and 2009. Clin Gastroenterol Hepatol 13: 1592-1600, 2015.

24. Jiang $\mathrm{W}$, Wu N, Wang $\mathrm{X}$, et al. Dysbiosis gut microbiota associated with inflammation and impaired mucosal immune function in intestine of human with non-alcoholic fatty liver disease. Sci Rep 5: 8096, 2015.

25. Imajo K, Fujita K, Yoneda M, et al. Hyperresponsivity to lowdose endotoxin during progression to nonalcoholic steatohepatitis in regulated by leptin-mediated signaling. Cell Metab 16: 44-54, 2012.

26. Saggioro A. Leaky gut, microbiota, and cancer: an incoming hypothesis. J Clin Gastroenterol 48: S62-S66, 2014.

27. Angulo P, Keach JC, Batts KP, Lindor KD. Independent predictors of liver fibrosis in patients with nonalcoholic steatohepatitis. Hepatology 30: 1356-1362, 1999.

The Internal Medicine is an Open Access journal distributed under the Creative Commons Attribution-NonCommercial-NoDerivatives 4.0 International License. To view the details of this license, please visit (https://creativecommons.org/licenses/ by-nc-nd/4.0/).

(C) 2019 The Japanese Society of Internal Medicine Intern Med 58: 2907-2913, 2019 